\title{
Characterisation of an ionic liquid-based gel polymer electrolyte for potential applications
}

\author{
K.W. Prasadini, K.S. Perera* and K.P. Vidanapathirana \\ Department of Electronics, Faculty of Applied Sciences, Wayamba University of Sri Lanka, Kuliyapitiya.
}

Submitted: 21 April 2018; Accepted: 28 September 2018

\begin{abstract}
Gel polymer electrolytes (GPEs) have received global attention as a viable substitute for liquid and solid electrolytes. They consist of a salt/solvent mixture encapsulated within a suitable polymer. GPEs have been immensely used for various applications. However, the presence of solvents has now been found to be creating some adverse effects for the performance of GPEs owing to their toxic features. Hence, attention has been focused on ionic liquids, which are known to be safe and have the ability to act as solvents. In this study, investigations were carried out on a gel polymer electrolyte prepared using 1-butyl-3-methylimidazolium trifluoromethanesulfonate as the ionic liquid, zinc trifluoromethanesulfonate as the salt and poly(vinylidinefluoride-co-hexafluoropropylene) as the polymer. It is well-known that ionic liquids are room temperature molten salts. They are considered as ionic solvents with plasticising effect. The optimum composition of the electrolyte, $14.2 \%$ polymer, $28.4 \%$ ionic liquid and $57.4 \%$ salt by weight showed a maximum room temperature conductivity of $4.52 \times 10^{-3} \mathrm{Scm}^{-1}$. This value together with the good mechanical properties proved the suitability of the GPE to be employed for energy storage applications. The sample was purely an ionic conductor. The electrochemical stability window was about $0.45 \mathrm{~V}$. It was observed that plating and stripping of $\mathrm{Zn}$ ions takes place on $\mathrm{Zn}$ electrodes but not on stainless steel electrodes.
\end{abstract}

Keywords: 1-butyl-3-methylimidazolium trifluoromethanesulfonate, electrochemical impedance spectroscopy, electrochemical stability window, ionic liquids, gel polymer electrolytes.

\section{INTRODUCTION}

Gel polymer electrolytes (GPEs) have been highlighted as excellent substitutes for liquid electrolytes for numerous applications such as batteries, super capacitors and fuel cells. A GPE consists of a polymer, a salt and solvents. It is assumed that the salt-solvent mixture is entrapped inside the polymer network. GPEs possess some distinct advantages over liquid electrolytes such as reduced leakage, improved safety and better shape flexibility. However, the daunting array of green environment related challenges motivated the scientists to find innovative approaches to substitute solvents of GPEs, which are known to be toxic for flora and fauna. Nonetheless, it has been found that these solvents are responsible for low thermal stability and poor electrochemical performance of GPEs (Pandey et al., 2011). As a result, there has been a rapid increase of global concern towards fabricating user-friendly GPEs.

Ionic liquids (ILs) have been considered for use as substitutes for organic solvents in the preparation of GPEs. They are also considered as ionic solvents with plasticising effects (Ramesh et al., 2014). Thus, a major research focus was aimed at ILs, which are present as room temperature molten salts. There are several unique features of ILs that make them a class of new eyecatching materials. Some of the key characteristics are high mechanical strength, good thermal stability, non toxicity, non flammability and high stability (Pandey et al., 2010). Besides, ILs assist conductivity in three ways; namely, i. by softening polymer backbone, ii. by weakening transient coordination bonds, and iii. by demolishing crystalline structure. The ILs have been successfully used with different polymers such as poly(vinylidinefluoride-co-hexafluoropropylene)

*Corresponding author (kumudu31966@gmail.com; (D) https://orcid.org/0000-0003-4491-662X)

This article is published under the Creative Commons CC-BY-ND License (http://creativecommons.org/licenses/by-nd/4.0/). This license permits use, distribution and reproduction, commercial and non-commercial, provided that the original work is properly cited and is not changed in anyway. 
(PVdF-co-HFP), poly(methylmethacrylate) (PMMA) and poly(vinylidine fluoride) (PVdF).

Among those, PVdF-co-HFP has received a growing interest due to its remarkable features. It is a semi-crystalline polymer having a superior mechanical integrity blended with a high dielectric constant and good electrochemical stability (Aravindan \& Vickraman, 2008; Miao et al., 2008). Also, the credits of low melting temperature and low glass transition temperature has made it a more convenient polymer host to be used in electrolytes. From a practical point of view, Li-based electrolyte systems have received a tremendous attraction and due to that, they have been immensely employed for diverse applications (Zhang et al., 2003). However, the hazardous nature of $\mathrm{Li}$ and high price have been identified as crucial facts today. Hence, there is a new insight into preparation, characterisation and application of non- $\mathrm{Li}$ based devices. Among them, $\mathrm{Zn}, \mathrm{Mg}$ and $\mathrm{Na}$ are some of the substitutes that have received a considerable recognition (Khoo et al., 2013; Weerasinghe et al., 2015). Subsequently, non-Li based electrolytes emerged as a new class of materials to be used for applications.

This paper reports a GPE based on an ionic liquid 1-butyl-3-methylimidazolium trifluoromethanesulfonate (1B3MITF) to be used for a rechargeable $\mathrm{Zn}$ battery. The polymer matrix of the present work is polyvinylidene fluoride (PVdF-co-HFP). Zinc trifluoromethanesulfonate $\left.\left[\mathrm{Zn}\left(\mathrm{CF}_{3} \mathrm{SO}_{3}\right)_{2}\right)-\mathrm{ZnTF}\right]$ has been used as the salt.

\section{METHODOLOGY}

\section{Preparation of the ionic liquid-based gel polymer electrolyte}

Poly (vinylidinfluoride-co-hexafluoropropylene) (PVdFco-HFP, average MW 400,000), 1-butyl-3-methylim idazoliumtrifluoromethanesulfonate (1B3MITF, 95 $\%)$ and zinc trifluoromethanesulfonate $\left[\mathrm{Zn}\left(\mathrm{CF}_{3} \mathrm{SO}_{3}\right)_{2}\right.$ ZnTF, $98 \%$ ] were used as received from Aldrich. ILbased GPE was prepared by traditional solvent casting technique. The required amount of PVdF-co-HFP was dissolved in acetone and it was magnetically stirred overnight. 1B3MITF and ZnTF were added according to the polymer content and magnetic stirring was continued overnight. The resulting homogeneous, clear, viscous liquid was poured into a well cleaned Petri dish and kept in a desiccator. Thereby, it was possible to obtain a bubble free, mechanically stable thin film.

\section{AC conductivity measurements of samples}

A circular shape sample was cut from the film and it was assembled in between two stainless steel (SS) electrodes in a spring-loaded sample holder. Impedance data were gathered in the frequency range, $0.1 \mathrm{~Hz}$ to $400 \mathrm{kHz}$ by using a computer controlled Metrohm Autolab Impedance Analyzer M101. Measurements were taken at room temperature for all samples and for the optimised sample, measurements were taken from room temperature to $55^{\circ} \mathrm{C}$. Thickness of the films were measured using a micrometer screw gauge.

\section{Transference number measurements}

A circular shaped sample was cut from the film with the optimised composition. It was sandwiched between two SS electrodes and assembled in a spring-loaded sample holder. A DC potential of $1 \mathrm{~V}$ was applied, and the drop of current was measured as a function of time by using a computer controlled Metrohm Autolab Potentiostat M101.

Determination of electrochemical stability window and plating- stripping effect

A sample was sandwiched between a SS electrode and a $\mathrm{Zn}$ electrode and assembled in a spring-loaded sample holder. Linear sweep voltammetry (LSV) study was performed in the potential range $-0.35 \mathrm{~V}$ to $0.32 \mathrm{~V}$ at the scan rate $5 \mathrm{mVs}^{-1}$ for the assembly of SS / GPE / $\mathrm{Zn}$. In the three-electrode electrochemical setup, SS electrode served as the working electrode, while the $\mathrm{Zn}$ electrode was used as counter and reference electrodes. A computer controlled Metrohm Autolab Potentiostat M101 was used to obtain the current variation with voltage. To study the plating stripping effect, cyclic voltammetry (CV) measurements were taken in the potential range $-0.5 \mathrm{~V}$ to $+0.5 \mathrm{~V}$ for assemblies with the configurations, SS/sample/SS and Zn/sample/Zn at the scan rate $10 \mathrm{mVs}^{-1}$.

\section{RESULTS AND DISCUSSION}

\section{Determination of the optimized composition at room temperature}

Impedance data were analysed using NOVA software dedicated to metrohm autolab impedance analyser. 


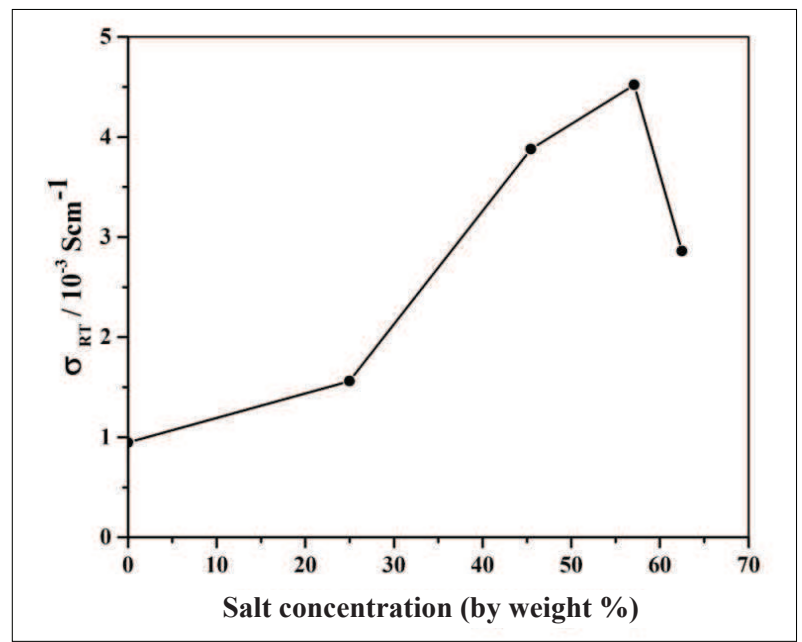

Figure 1: Room temperature ionic conductivity variation with salt concentration (by weight percentage)

Conductivity $(\sigma)$ was calculated using the following equation;

$\sigma=\left(1 / R_{b}\right)(t / A)$

where $R_{b}$ is the bulk electrolyte resistance, $t$ is the thickness of the electrolyte and A is the area of the electrolyte.

Figure 1 shows the variation of room temperature conductivities $\left(\sigma_{\mathrm{RT}}\right)$ with the salt concentration (weight percentage - wt.\%).

The conductivity values increased with increasing salt concentration. It reached a maximum value of $4.52 \times 10^{-3} \mathrm{Scm}^{-1}$ and then decreased. These observations reveal that there should be an interplay between some factors administrating the conductivity behaviour with the salt concentration.

Ionic conductivity, $\sigma$, greatly depends on two factors, namely, ion concentration (n) and ion mobility ( $\mu$ ) as follows;

$\sigma=n e \mu$

where e is the charge of an ion. One possible reason for the initial enhancement of ionic conductivity is the increase of the concentration of mobile charge species with the increase of salt concentration. The decrease in ion conductivity was observed after 57.4 wt. \% of ZnTF.

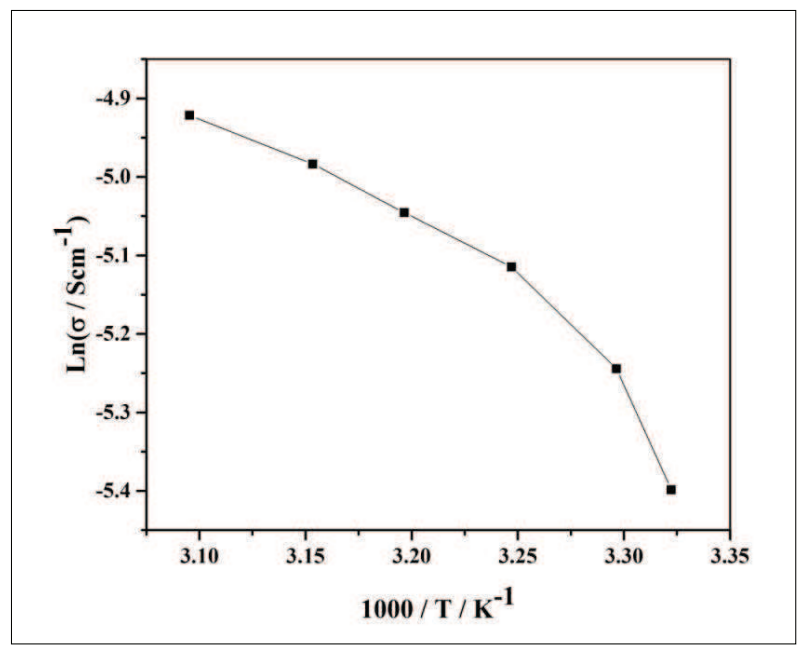

Figure 2: Temperature dependence ionic conductivity of IL based GPE with the composition $14.2 \%$ PVdF-co-HFP, $28.4 \% 1 \mathrm{~B} 3 \mathrm{MITF}$ and $57.1 \% \mathrm{Zn}\left(\mathrm{CF}_{3} \mathrm{SO}_{4}\right)_{2}$. Film thickness $=0.28 \mathrm{~mm}$ and area $=1.54 \mathrm{~cm}^{2}$

It may be due to the dominant effect of viscosity of the medium that limits the ionic mobility. Further, at higher salt concentrations, the concentration of the mobile charge species decrease due to the formation of ion agglomerates (Ravindran \& Vickraman, 2012), which do not assist the conductivity.

\section{Temperature dependence of conductivity}

Conductivity variation with the temperature for the optimised electrolyte is shown in Figure 2. There are two clear features in the graph:

i. It follows the Vogel-Tamman-Fulcher (VTF) behaviour throughout the temperature variation.

VTF behaviour can be given by the following equation;

$\sigma=\mathrm{AT}^{-1 / 2} \exp \left(\mathrm{E}_{\mathrm{a}} / \mathrm{k}_{\mathrm{B}}\left(\mathrm{T}-\mathrm{T}_{0}\right)\right.$

Here, $\mathrm{A}$ is the pre exponential factor, $\mathrm{E}_{\mathrm{a}}$ is the activation energy, $k_{B}$ is the Boltzmann constant and $T_{0}$ is the equilibrium glass transition temperature. Most of the IL based GPEs are showing this behaviour (Tafur \& Romero, 2014). When a system obeys the VTF behaviour, it is said to be depending on free volume theory. That is, the mobility of the mobile charged species is governed by polymer segmental motion by providing sufficient free space. 
ii. Ionic conductivity increases with increasing temperature

With temperature increment, viscosity goes down. This leads to increase the mobility of the charged species and the ionic conductivity. In literature, there are some reports explaining this as due to the hopping mechanism between coordinating sites, local structural relaxations and segmental motion of the polymer (Jayathilake et al., 2014). Further, when the temperature is increased, ions get energised and the mobility increases. As a result, conductivity increases. In addition to that, polymer network expands easily upon increase in temperature giving rise to free volume. This leads to increase in ion mobility and it contributes to conductivity immensely (Polu et al., 2012).

\section{Transference number measurements}

Total ionic transference number $\left(\mathrm{t}_{\text {ion }}\right)$ was determined using the DC polarisation test. The resulting graph is shown in Figure 3.

$t_{\text {ion }}$ was calculated according to the equation,

$\mathrm{t}_{\text {ion }}=\left(\mathrm{i}_{\mathrm{i}}-\mathrm{i}_{0}\right) / \mathrm{i}_{\mathrm{i}}$

where $\mathrm{i}_{\mathrm{i}}$ is the initial current and $\mathrm{i}_{0}$ is the stable current.

Here, SS electrodes are blocking ions. At the beginning, an abrupt reduction of current was observed due to polarising of ions. Thereafter, a residual constant current

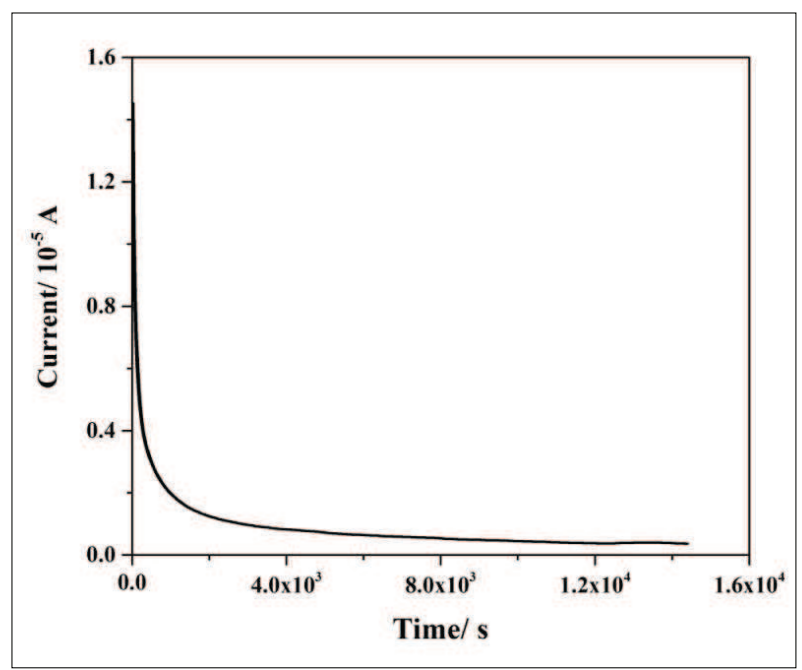

Figure 3: DC polarisation graph for the symmetric cell with SS electrodes under a DC bias potential of $1 \mathrm{~V}$ arises as a result of electrons, which are not blocked by the electrodes.

According to the above equation, $\mathrm{t}_{\text {ion }}$ of the optimised sample was approximately 0.95 . This predicts that overall conductivity of the IL-based GPE is predominantly ionic. The electronic contribution is negligible (Pandey et al., 2009 ; Kumar \& Hashmi, 2010).

\section{Determination of electrochemical stability window and plating- stripping effect}

Accurate determination of electrochemical stability window of a GPE is extremely important from a practical point of view. It measures the ability of the components of a GPE to resist oxidative and reductive decomposition on electrodes. When the respective redox processes are in progress, the electrolyte should be in inert state (Cheng et al., 2012). By using linear sweep voltammogram, it can predict the endure potential range for the electrolyte (Deraman et al., 2013). Figure 4 shows the linear sweep voltammogram for the cell with SS/IL-based GPE/Zn at the scan rate of $5 \mathrm{mVs}^{-1}$. From Figure 4 it is obvious that the tolerable operating voltage range for the cell with ILbased GPE is $-0.25 \mathrm{~V}$ to $0.2 \mathrm{~V}$. This width of the stability window is about $0.45 \mathrm{~V}$. For small scale devices, this value is acceptable. The narrow stability window can be due to IL, which is entrapped in PVdF-co-HFP to form the GPE (Pandey \& Hashmi, 2013). This value is quite smaller than the value reported previously for a PVdFCo-HFP/ZnTF/1E3MITF system (Xu et al., 2005). One possible reason may be the difference of ionic liquid

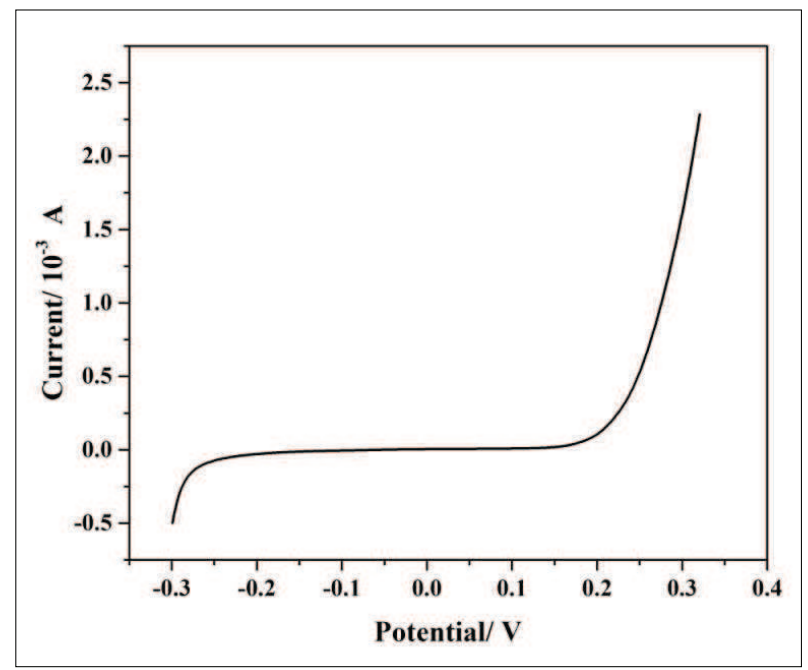

Figure 4: Linear sweep voltammogram for the cell with SS/IL-based $\mathrm{GPE} / \mathrm{Zn}$ at scan rate of $5 \mathrm{mVs}^{-1}$. 
type. However, further studies are underway to increase the electrochemcial stability window.

The cyclic voltammograms for the cells SS/IL based GPE/SS and Zn/IL based GPE/Zn are shown in Figure 5. Anodic and cathodic peaks are not observed for the assembly with SS electrodes, but they are visible with $\mathrm{Zn}$ electrodes. This elucidates the fact that $\mathrm{Zn}$ ion plating and stripping is facile only on Zn electrodes (Jayathilake et al., 2014). Similar observations have been reported previously (Kumar et al., 2011; Tafur et al., 2015). Peak current densities of $\mathrm{Zn}$ plating and stripping are about $0.06 \mathrm{~A}$. This value is promising for the GPE to be used for applications.

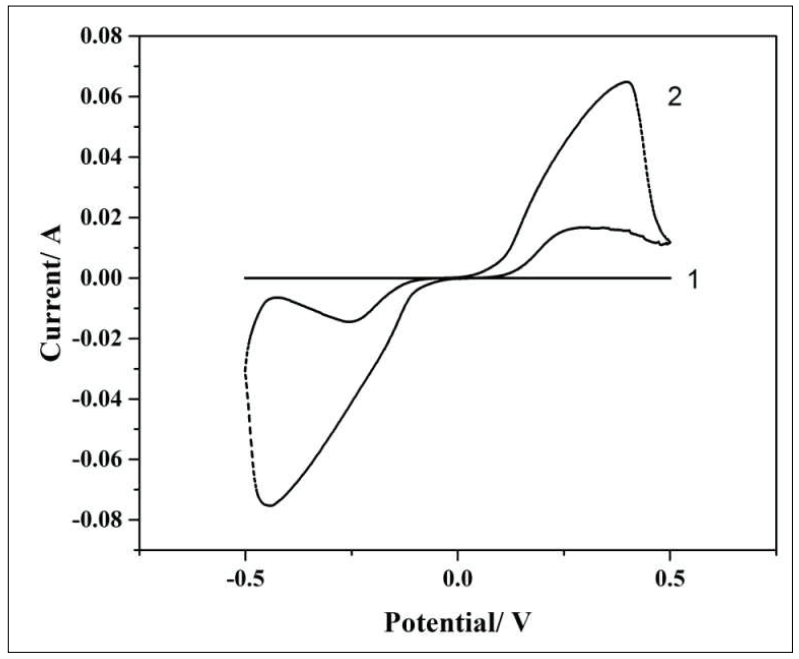

Figure 5: Cyclic voltammograms for the cells (1) SS/IL based GPE/SS and (2) $\mathrm{Zn} / \mathrm{IL}$ based GPE/Zn at scan rate of $10 \mathrm{mVs}^{-1}$

\section{CONCLUSION}

An IL-based GPE with 1B3MITF, ZnTF and PVdF-coHFP was prepared as a mechanically stable, free standing film using the solvent casting technique. The optimised composition was $14.2 \%$ PVdF-co-HFP, $28.4 \%$ 1B3MITF and $57.4 \% \mathrm{ZnTF}$. It has a room temperature conductivity of $4.52 \times 10^{-3} \mathrm{Scm}^{-1}$. This is an acceptable value for applications. The conduction mechanism is based on free volume theory. The sample is a pure ionic conductor. $\mathrm{Zn}$ ion plating and stripping takes place only on Zn electrodes but not on SS electrodes. This is a key attribute to use this GPE for Zn-based applications.

\section{Acknowledgement}

Authors wish to acknowledge the National Science Foundation of Sri Lanka for financial support under the research grants, RG/2015/EQ/07 and RG/2017/BS/02.

\section{REFERENCES}

Aravindan P. \& Vickraman P. (2008). Polyvinylidenefluoride hexafluoropropylene based nano composite polymer electrolytes (NCPE) complexed with $\mathrm{LiPF}_{3}\left(\mathrm{CF}_{3} \mathrm{SO}_{2}\right)_{3}$. European Polymer Journal 43: 5121-5127.

DOI: https://doi.org/10.1016/j.eurpolymj.2007.10.003

Cheng J., Wen Y., Cao G., Yang Y. \& Liu H. (2012). Impedance study of electrochemical stability limits for electrolytes. International Journal of Electrochemical Science 7: 12383-12390.

Deraman S.K., Mohamed N.S. \& Subban R.H.Y. (2013). Conductivity and electrochemical studies on polymer electrolytes based on poly vinyl (chloride)-ammonium triflate-ionic liquid for proton battery. International Journal of Electrochemical Science 8: 1459-1468.

Jayathilake Y.M.C.D., Perera K.S., Vidanapathirana K.P. \& Bandara L.R.A.K. (2014). A novel gel polymer electrolyte based on polymethylmethacrylate and copper trifluoromethanesulfonate. Journal of Electroanalytical Chemistry 724: 125-129.

DOI: https://doi.org/10.1016/j.jelechem.2014.04.018

Khoo T., Somers A., Torriero A.A.J., MacFarlane D.R., Howlett P.C. \& Forsyth M. (2013). Discharge behaviour and interfacial properties of a magnesium battery incorporating trihexyl (tetradecyl) phosphonium based ionic liquid electrolytes. Electrochimica Acta 87: 701-708. DOI: https://doi.org/10.1016/j.electacta.2012.09.072

Kumar D. \& Hashmi S.A. (2010). Ionic liquid based sodium ion conducting gel polymer electrolytes. Solid State Ionics 181: 416-423.

DOI: https://doi.org/10.1016/j.ssi.2010.01.025

Kumar Y., Hashmi S.A. \& Pandey G.P. (2011). Ionic liquid mediated $\mathrm{Mg}$ ion conduction in $\mathrm{PEO}$ based polymer electrolytes. Electrochimica Acta 56: 3864-3873. DOI: https://doi.org/10.1016/j.electacta.2011.02.035

Miao R., Liu B., Zhu Z., Liu Y., Li J., Wang X. \& Li Q. (2008). PVdF HFP based porous electrolyte membranes for Li ion batteries. Journal of Power Sources 184: 420-426. DOI: https://doi.org/10.1016/j.jpowsour.2008.03.045

Pandey G.P., Agrawal R.C. \& Hashmi S.A. (2009). Magnesium ion-conducting gel polymer electrolytes dispersed with nanosized magnesium oxide. Journal of Power Sources 190: $563-572$.

DOI: https://doi.org/10.1016/j.jpowsour.2009.01.057

Pandey G.P., Kumar Y. \& Hashmi S.A. (2010). Ionic liquid incorporated polymer electrolytes for supercapacitor application. Indian Journal of Chemistry 49A:743-751. 
Pandey G.P., Kumar Y. \& Hashmi S.A. (2011). Ionic liquid incorporated PEO based polymer electrolyte for electrical double layer capacitors: a comparative study with lithium and magnesium systems. Solid State Ionics 190: 93-98. DOI: https://doi.org/10.1016/j.ssi.2011.03.018

Pandey G.P. \& Hashmi S.A. (2013). Ionic liquid 1-ethyl-3methylimidazolium tetracyanoborate-based gel polymer electrolyte for electrochemical capacitors. Journal of Materials Chemistry A 10: 3372-3378.

Polu A.R., Kumar R. \& Kumar K.V. (2012). Ionic conductivity and electrochemical cell studies of new $\mathrm{Mg}^{2+}$ ion conducting PVA/PEG based polymer blend electrolytes. Advanced Material Letters 3(5): 406-409.

Ramesh S., Uma O., Shanti R., Yi L.J. \& Ramesh K. (2014). Preparation and characterization of poly (ethyl methacrylate) based polymer electrolytes doped with 1-butyl-3-methylimidazolium trifluoromethanesulfonate. Measurement 48: 263-273.

DOI: https://doi.org/10.1016/j.measurement.2013.11.025

Ravindran D. \& Vickraman P. (2012). $\mathrm{Mg}^{2+}$ ionic conductivity behavior of mixed salt system in PVA-PEG blend matrix. International Journal of Scientific and Research
Publications 2(12): 1-4.

Tafur J.P. \& Romero A.J.F. (2014). Electrical and spectroscopic characterization of PVdF-HFP and TFSI-ionic liquidsbased gel polymer electrolyte membranes. Influence of ZnTf2 salt. Journal of Membrane Science 469: 499-506. DOI: https://doi.org/10.1016/j.memsci.2014.07.007

Tafur J.P., Santos F. \& Romero A.J.F. (2015). Influence of the ionic liquid type on the gel polymer electrolytes properties. Membranes 5: 752-771.

DOI: https://doi.org/10.3390/membranes5040752

WeerasingheW.A.D.S.S., Madhushani L.K.M., Vidanapathirana K.P. \& Perera K.S. (2015). Performance of Zn rechargeable cells having polypyrrole cathodes doped with surfactant anion. Ruhuna Journal of Science 6: 43-50.

$\mathrm{Xu}$ J.J., Ye H. \& Huang J. (2005). Novel zinc ion conducting polymer electrolytes based on ionic liquids. Electrochemistry Communications 7: 1309-1317. DOI: https://doi.org/10.1016/j.elecom.2005.09.011

Zhang S.S., Xu K. \& Jow T.R. (2003). Li ion cell with poly(acrylonitrile-methyl methacrylate) based gel polymer electrolyte. Solid State Ionics 158: 375-380.

DOI: https://doi.org/10.1016/S0167-2738(02)00914-1 